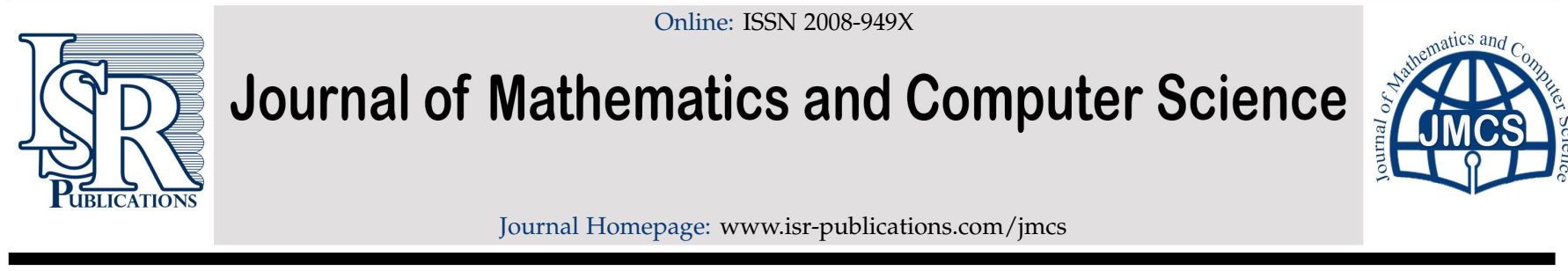

\title{
Generalized essential maps and coincidence type theory for compact multifunctions
}

\author{
Donal O'Regan
}

School of Mathematics, Statistics and Applied Mathematics, National University of Ireland, Galway, Ireland.

\begin{abstract}
In this paper we discuss generalized essential maps. By establishing a very simple result we are able to present a variety of topological transversality theorems in a general setting
\end{abstract}

Keywords: Essential maps, homotopy, admissible maps.

2020 MSC: 47H10, 54H25, 55M20.

(C)2020 All rights reserved.

\section{Introduction}

The topological transversality theorem [4] for continuous compact maps states that for continuous compact maps $F$ and $G$ with $F \cong G$ then $F$ is essential if and only if $G$ is essential. The essential map theory was extended to set valued maps and to d-essential maps [6-8]. In this paper we consider admissible maps (see below) and we establish a very general topological transversality theorem. To do this we first present a very simple result which we will then use to establish topological transversality theorems in a variety of settings.

Let $X, Y$ be metric spaces and $\Gamma$ paracompact. A continuous single valued map $p: \Gamma \rightarrow X$ is called a Vietoris map (written $p: \Gamma \Rightarrow X$ ) if the following two conditions are satisfied:

(i). for each $x \in X$, the set $p^{-1}(x)$ is acyclic (with respect to the Čech cohomology functor), (ii). $p$ is a perfect map i.e., $p$ is closed and for every $x \in X$ the set $p^{-1}(x)$ is nonempty and compact.

Let $D(X, Y)$ be the set of all admissible pairs $X \stackrel{p}{\models} \Gamma \stackrel{q}{\rightarrow} Y$ where $p$ is a Vietoris map and $q$ is continuous. We will denote every such diagram by $(p, q)$. Given two diagrams $(p, q)$ and $\left(p^{\prime}, q^{\prime}\right)$, where $\mathrm{X} \stackrel{p^{\prime}}{\Leftarrow} \Gamma^{\prime} \stackrel{\mathrm{q}^{\prime}}{\rightarrow} Y$, we write $(\mathrm{p}, \mathrm{q}) \sim\left(\mathrm{p}^{\prime}, \mathrm{q}^{\prime}\right)$ if there a homeomorphism $\mathrm{f}: \Gamma \rightarrow \Gamma^{\prime}$ such that $\mathrm{p}^{\prime} \circ \mathrm{f}=\mathrm{p}$ and $q^{\prime} \circ f=q$. The equivalence class of a diagram $(p, q) \in D(X, Y)$ with respect to $\sim$ is denoted by

$$
\phi=\{\mathrm{X} \stackrel{p}{\models} \Gamma \stackrel{\mathrm{q}}{\rightarrow} \mathrm{Y}\}: \mathrm{X} \rightarrow \mathrm{Y}
$$

Email address: donal . oregan@nuigalway.ie (Donal O’Regan)

doi: $10.22436 /$ jmcs.021.02.02

Received: 2019-09-05 Revised: 2019-10-22 Accepted: 2020-03-03 
or $\phi=[(p, q)]$ and is called a morphism from $X$ to $Y$. We let $M(X, Y)$ be the set of all such morphisms. Note if $(p, q),\left(p_{1}, q_{1}\right) \in D(X, Y)$ (where $X \stackrel{p}{\models} \Gamma \stackrel{q}{\rightarrow} Y$ and $X \stackrel{p_{1}}{\models} \Gamma^{\prime} \stackrel{q_{1}}{\rightarrow} Y$ ) and $(p, q) \sim\left(p_{1}, q_{1}\right)$ then it is easy to see that for $x \in X$ we have $q_{1}\left(p_{1}^{-1}(x)\right)=q\left(p^{-1}(x)\right)$. For any $\phi \in M(X, Y)$ a set $\phi(x)=$ $q p^{-1}(x)$ where $\phi=[(p, q)]$ is called an image of $x$ under a morphism $\phi$. Let $\phi \in M(X, Y)$ and $(p, q)$ a representative of $\phi$. We define $\phi(X) \subseteq Y$ by $\phi(X)=q\left(p^{-1}(X)\right)$. Note $\phi(X)$ does not depend on the representative of $\phi$. Now $\phi \in M(X, Y)$ is called compact provided the set $\phi(X)$ is relatively compact in $Y$. We say a map $\phi$ is admissible or determined by a morphism $\{X \stackrel{p}{\models} \Gamma \stackrel{q}{\rightarrow} Y\}$ provided $\phi(x)=q p^{-1}(x)$ for any $x \in X$ and we write $\phi \in \operatorname{Adm}(X, Y)$ (note $\phi$ is upper semicontinuous) i.e., $\operatorname{Adm}(X, Y)$ denotes the class of all admissible set-valued maps $\phi: X \rightarrow 2^{Y}$ (note a set-valued map $\phi: X \rightarrow 2^{Y}$ is admissible if it is represented by an admissible pair). Let $U$ be open in $X$ and let $F, G \in \operatorname{Adm} \partial u(\bar{U}, X)$ (i.e., $F, G \in$ $\operatorname{Adm}(\bar{U}, X)$ with $x \notin F(x), x \notin G(x)$ for $x \in \partial U$ ) be compact maps. We say $F \cong G$ (compactly) in $\operatorname{Adm}_{\partial \mathrm{u}}(\overline{\mathrm{U}}, \mathrm{X})$ if there exists a (compact) admissible $\Psi: \overline{\mathrm{U}} \times[0,1] \rightarrow 2^{\mathrm{X}}$ with $\mathrm{x} \notin \Psi_{\mathrm{t}}(\mathrm{x})$ for any $\mathrm{x} \in \partial \mathrm{U}$ and $t \in(0,1), \Psi_{0}=F$ and $\Psi_{1}=\mathrm{G}$ (here $\left.\Psi_{\mathrm{t}}(\mathrm{x})=\Psi(x, \mathrm{t})\right)$. Note $\cong$ (compactly) in Admau $(\overline{\mathrm{U}}, \mathrm{X})$ is an equivalence relation; see [3, Section 46], [5, Section 5]. Suppose $F \in \operatorname{Adm} \partial u(\bar{U}, X)$ is a compact map and $f: \bar{U} \rightarrow X$ is a single valued continuous compact map with $x \neq f(x)$ for $x \in \partial U$. For a condition (clearly satisfied if $f$ is the zero map) to guarantee that $F \cong f$ (compactly) in $\operatorname{Adm} \partial u(\bar{U}, X)$ see [3, (Section 46), Proposition 46.3].

\section{Topological Transversality Theorem}

We will consider classes $\mathbf{A}$ and $\mathbf{B}$ of maps. Let $E$ be a completely regular space and $U$ an open subset of $E$.

Definition 2.1. We say $F \in A(\bar{U}, E)$ if $F \in \mathbf{A}(\bar{U}, E)$ and $F: \bar{U} \rightarrow K(E)$ is a upper semicontinuous (u.s.c.) compact map; here $\bar{U}$ denotes the closure of $U$ in $E$ and $K(E)$ denotes the family of nonempty compact subsets of $E$.

Remark 2.2. Examples of $F \in \mathbf{A}(\bar{U}, E)$ might be that $F$ has convex values or $F$ has acyclic values or $F$ is admissible (as described in Section 1).

In this paper we fix a $\Phi \in B(\bar{U}, E)$ (i.e., $\Phi \in \mathbf{B}(\bar{U}, E)$ and $\Phi: \bar{U} \rightarrow K(E)$ is a u.s.c. map).

Definition 2.3. We say $F \in A_{\partial u}(\bar{U}, E)$ if $F \in A(\bar{U}, E)$ and $\Phi(x) \cap F(x)=\emptyset$ for $x \in \partial U$; here $\partial U$ denotes the boundary of $\mathrm{U}$ in $\mathrm{E}$.

Definition 2.4. Let $F, G \in A_{\partial u}(\bar{U}, E)$. We say $F \cong G$ in $A_{\partial u}(\bar{U}, E)$ if there exists a u.s.c. compact map $\Psi: \overline{\mathrm{U}} \times[0,1] \rightarrow \mathrm{K}(\mathrm{E})$ with $\Psi \in \mathbf{A}(\overline{\mathrm{U}} \times[0,1], \mathrm{E}), \Phi(x) \cap \Psi_{\mathrm{t}}(\mathrm{x})=\emptyset$ for any $x \in \partial \mathrm{U}$ and $\mathrm{t} \in(0,1)$ (here $\left.\Psi_{\mathrm{t}}(\mathrm{x})=\Psi(\mathrm{x}, \mathrm{t})\right), \Psi_{0}=\mathrm{F}$ and $\Psi_{1}=\mathrm{G}$. In addition here we always assume for any map $\Theta \in \mathbf{A}(\overline{\mathrm{U}} \times[0,1], \mathrm{E})$ and any maps $g \in \mathbf{C}(\overline{\mathrm{u}}, \overline{\mathrm{u}} \times[0,1])$ and $f \in \mathbf{C}(\overline{\mathrm{U}} \times[0,1], \overline{\mathrm{u}} \times[0,1])$ then $\Theta \circ g \in \mathbf{A}(\overline{\mathrm{u}}, \mathrm{E})$ and $\Theta \circ f \in$ $\mathbf{A}(\overline{\mathrm{U}} \times[0,1], \mathrm{E})$; here $\mathbf{C}$ denotes the class of single valued continuous functions.

\section{Remark 2.5.}

(a). In our results below alternatively we could use the following definition for $\cong$ in $A_{\partial u}(\bar{U}, E): F \cong G$ in $A_{\partial u}(\bar{U}, E)$ if there exists a u.s.c. compact map $\Psi: \bar{U} \times[0,1] \rightarrow K(E)$ with $\Psi(., \eta().) \in \mathbf{A}(\bar{U}, E)$ for any continuous function $\eta: \bar{u} \rightarrow[0,1]$ with $\eta(\partial U)=0, \Phi(x) \cap \Psi_{t}(x)=\emptyset$ for any $x \in \partial U$ and $t \in(0,1)$ (here $\left.\Psi_{\mathrm{t}}(\mathrm{x})=\Psi(\mathrm{x}, \mathrm{t})\right), \Psi_{0}=\mathrm{F}$ and $\Psi_{1}=\mathrm{G}$. [Note the additional assumption in Definition 2.4 is not needed here].

(b). Throughout the paper we assume $\cong$ in $A_{\partial u}(\bar{U}, E)$ is a reflexive, symmetric relation.

Remark 2.6. Let $F \in A_{\partial u}(\bar{U}, E)$. We say $F$ is $\Phi$-essential in $A_{\partial u}(\bar{U}, E)$ if for every map $J \in A_{\partial u}(\bar{U}, E)$ with $\left.\mathrm{J}\right|_{\partial \mathrm{u}}=\left.\mathrm{F}\right|_{\partial \mathrm{u}}$ and $\mathrm{J} \cong \mathrm{F}$ in $A_{\partial \mathrm{u}}(\overline{\mathrm{U}}, \mathrm{E})$ there exists a $\mathrm{x} \in \mathrm{U}$ with $\Phi(\mathrm{x}) \cap \mathrm{J}(\mathrm{x}) \neq \emptyset$.

We now present a simple result which will more or less immediately yield a very general topological transversality theorem. 
Theorem 2.7. Let $\mathrm{E}$ be a completely regular topological space, $\mathrm{U}$ an open subset of $\mathrm{E}, \mathrm{F} \in \mathrm{A}_{\partial \mathrm{u}}(\overline{\mathrm{U}}, \mathrm{E})$ and $\mathrm{G} \in \mathrm{A}_{\partial \mathrm{u}}(\overline{\mathrm{U}}, \mathrm{E})$ is $\Phi$-essential in $\mathrm{A}_{\partial \mathrm{u}}(\overline{\mathrm{U}}, \mathrm{E})$. Also suppose

$$
\left\{\begin{array}{l}
\text { for any map } \mathrm{J} \in \mathrm{A}_{\partial \mathrm{u}}(\overline{\mathrm{U}}, \mathrm{E}) \text { with }\left.\mathrm{J}\right|_{\partial \mathrm{u}}=\left.\mathrm{F}\right|_{\partial \mathrm{u}} \text { and } \\
\mathrm{J} \cong \mathrm{F} \text { in } \mathrm{A}_{\partial \mathrm{u}}(\overline{\mathrm{U}}, \mathrm{E}) \text { we have } \mathrm{G} \cong \mathrm{J} \text { in } \mathrm{A}_{\partial \mathrm{u}}(\overline{\mathrm{U}}, \mathrm{E})
\end{array}\right.
$$

Then $\mathrm{F}$ is essential in $\mathrm{A}_{\partial \mathrm{u}}(\overline{\mathrm{U}}, \mathrm{E})$.

Proof. Without loss of generality assume $\cong$ in $A_{\partial u}(\bar{U}, E)$ is as in Definition 2.4. Consider any map $J \in$ $A_{\partial u}(\bar{U}, E)$ with $\left.J\right|_{\partial u}=\left.F\right|_{\partial u}$ and $J \cong F$ in $A_{\partial u}(\bar{U}, E)$. From (2.1) there exists a u.s.c. compact map $H^{J}: \bar{U} \times[0,1] \rightarrow K(E)$ with $H^{J} \in \mathbf{A}(\bar{U} \times[0,1], E), \Phi(x) \cap H_{t}^{J}(x)=\emptyset$ for any $x \in \partial U$ and $t \in(0,1)$ (here $\left.H_{t}^{J}(x)=H^{J}(x, t)\right), H_{0}^{J}=G$ and $H_{1}^{J}=J$. Let

$$
\mathrm{K}=\left\{x \in \overline{\mathrm{U}}: \Phi(\mathrm{x}) \cap \mathrm{H}^{\mathrm{J}}(\mathrm{x}, \mathrm{t}) \neq \emptyset \text { for some } \mathrm{t} \in[0,1]\right\}
$$

and

$$
\mathrm{D}=\left\{(\mathrm{x}, \mathrm{t}) \in \overline{\mathrm{U}} \times[0,1]: \Phi(\mathrm{x}) \cap \mathrm{H}^{\mathrm{J}}(\mathrm{x}, \mathrm{t}) \neq \emptyset\right\} .
$$

Now $\mathrm{D} \neq \emptyset$ (note $\mathrm{G}$ is $\Phi$-essential in $A_{\partial \mathrm{u}}(\overline{\mathrm{U}}, \mathrm{E})$ ) and $\mathrm{D}$ is closed (note $\Phi$ and $\mathrm{H}^{\mathrm{J}}$ are u.s.c.) and so $\mathrm{D}$ is compact (note $\mathrm{H}^{\mathrm{J}}$ is a compact map). Let $\pi: \overline{\mathrm{U}} \times[0,1] \rightarrow \overline{\mathrm{U}}$ be the projection. Now $\mathrm{K}=\pi(\mathrm{D})$ is closed (see Kuratowski's theorem [2, pp 126]) and so in fact compact (recall projections are continuous). Also note $\mathrm{K} \cap \partial \mathrm{U}=\emptyset$ (since $\Phi(x) \cap \mathrm{H}_{\mathrm{t}}^{\mathrm{t}}(\mathrm{x})=\emptyset$ for any $\mathrm{x} \in \partial \mathrm{U}$ and $\mathrm{t} \in[0,1]$ ) so since $\mathrm{E}$ is Tychonoff there exists a continuous map $\mu: \bar{U} \rightarrow[0,1]$ with $\mu(\partial \mathrm{U})=0$ and $\mu(\mathrm{K})=1$. Define the map $\mathrm{R}$ by $\mathrm{R}(\mathrm{x})=\mathrm{H}^{\mathrm{J}}(\mathrm{x}, \mu(\mathrm{x}))$. Now $\mathrm{R} \in \mathrm{A}_{\partial \mathrm{u}}(\overline{\mathrm{U}}, \mathrm{E})$ (note $\mathrm{H}^{\mathrm{J}}(\mathrm{x}, \mu(\mathrm{x}))=\mathrm{H}^{\mathrm{J}} \circ \mathrm{g}(\mathrm{x})$ where $\mathrm{g}: \overline{\mathrm{U}} \rightarrow \overline{\mathrm{U}} \times[0,1]$ is given by $g(x)=(x, \mu(x))$ ) with $\left.R\right|_{\partial u}=\left.G\right|_{\partial u}$ (note if $x \in \partial U$ then $R(x)=H^{J}(x, 0)=G(x)$ and so $R(x) \cap \Phi(x)=G(x) \cap \Phi(x))$. We now show $R \cong G$ in $A_{\partial u}(\bar{U}, E)$. To see this let $Q: \bar{U} \times[0,1] \rightarrow K(E)$ be given by $Q(x, t)=H^{J}(x, t \mu(x))=H^{J} \circ f(x, t)$ where $f: \bar{U} \times[0,1] \rightarrow \bar{U} \times[0,1]$ is given by $f(x, t)=(x, t \mu(x))$. Note $Q \in \mathbf{A}(\overline{\mathrm{U}} \times[0,1], E), Q_{0}=G, Q_{1}=R$ and $\Phi(x) \cap Q_{t}(x)=\emptyset$ for any $x \in \partial U$ and $t \in(0,1)$ (since if $\mathrm{t} \in(0,1)$ and $\mathrm{x} \in \partial \mathrm{U}$ then $\Phi(\mathrm{x}) \cap \mathrm{H}^{\mathrm{J}}(\mathrm{x}, \mathrm{t} \mu(\mathrm{x}))=\Phi(\mathrm{x}) \cap \mathrm{H}_{\mathrm{t} \mu(\mathrm{x})}^{\mathrm{J}}(\mathrm{x})$ so $\mathrm{x} \in \mathrm{K}$ and as a result $\mu(\mathrm{x})=1$ i.e., $\left.\Phi(x) \cap H^{J}(x, t \mu(x))=\Phi(x) \cap H^{J}(x, t)\right)$. Thus $R \cong G$ in $A_{\partial u}(\bar{U}, E)$. Since $G$ is $\Phi$-essential in $A_{\partial u}(\bar{U}, E)$ there exists a $x \in U$ with $\Phi(x) \cap R(x) \neq \emptyset$ (i.e., $\left.\Phi(x) \cap H_{\mu(x)}^{J}(x) \neq \emptyset\right)$. Thus $x \in K, \mu(x)=1$ and so $\emptyset \neq \Phi(x) \cap H_{1}^{\mathrm{J}}(x)=\Phi(x) \cap \mathrm{J}(\mathrm{x})$.

\section{Remark 2.8.}

(i). In the proof of Theorem 2.7 it is simple to adjust the proof if we use $\cong$ in $A_{\partial u}(\bar{U}, E)$ from Remark 2.5 if we note $\mathrm{R}()=.\mathrm{H}^{\mathrm{J}}(., \mu()$.$) and \mathrm{Q}(., v())=.\mathrm{H}^{\mathrm{J}}(., v(.) \mu())=.\mathrm{H}^{\mathrm{J}}(., w()$.$\left.) (with w()=.v(.) \mu().\right)$ for any continuous $v: \overline{\mathrm{U}} \rightarrow[0,1]$ with $v(\partial \mathrm{U})=0$ (note $w: \overline{\mathrm{U}} \rightarrow[0,1]$ is continuous and $w(\partial \mathrm{U})=0$ ).

(ii). One could replace u.s.c. in the definition of $A(\bar{U}, E), B(\bar{U}, E)$, Definition 2.4 and Remark 2.5 with any condition that guarantees that $\mathrm{K}$ in the proof of Theorem 2.7 is closed; this is all that is needed if $E$ is normal. If $E$ is Tychonoff and not normal the one can also replace the compactness of the map in $A(\bar{U}, E)$, Definition 2.4 and Remark 2.5 with any condition that guarantees that $\mathrm{K}$ in the proof of Theorem 2.7 is compact.

Example 2.9. Theorem 2.7 immediately yields a general Leray-Schauder type alternative for coincidences. Let $E$ be a completely metrizable locally convex space, $U$ an open subset of $E, F \in A_{\partial u}(\bar{U}, E), G \in$ $A_{\partial u}(\bar{U}, E)$ is $\Phi$-essential in $A_{\partial u}(\bar{U}, E)$ and $\Phi(x) \cap[t F(x)+(1-t) G(x)]=\emptyset$ for $x \in \partial U$ and $t \in(0,1)$. For any map $J \in A_{\partial u}(\bar{U}, E)$ with $\left.J\right|_{\partial u}=\left.F\right|_{\partial u}$ suppose $H^{J} \in \mathbf{A}(\bar{U} \times[0,1], E)$ where $H^{J}(x, t)=t J(x)+(1-$ t) $G(x)$ [Also here we assume for any map $\Theta \in \mathbf{A}(\bar{U} \times[0,1], E)$ and any maps $g \in \mathbf{C}(\bar{U}, \bar{U} \times[0,1])$ and $f \in \mathbf{C}(\overline{\mathrm{u}} \times[0,1], \overline{\mathrm{u}} \times[0,1])$ then $\Theta \circ \mathrm{g} \in \mathbf{A}(\overline{\mathrm{U}}, \mathrm{E})$ and $\Theta \circ f \in \mathbf{A}(\overline{\mathrm{U}} \times[0,1], E)]$. Then $\mathrm{F}$ is $\Phi$-essential in $A_{\partial u}(\bar{U}, E)$. 
The proof follows from Theorem 2.7 since topological vector spaces are completely regular and note if $\mathrm{J} \in \mathrm{A}_{\partial \mathrm{u}}(\overline{\mathrm{U}}, \mathrm{E})$ with $\left.\mathrm{J}\right|_{\partial \mathrm{u}}=\left.\mathrm{F}\right|_{\partial u}$ then with $\mathrm{H}^{\mathrm{J}}(\mathrm{x}, \mathrm{t})=\mathrm{t} J(\mathrm{x})+(1-\mathrm{t}) \mathrm{G}(\mathrm{x})$ note $\mathrm{H}_{0}^{\mathrm{J}}=\mathrm{G}, \mathrm{H}_{1}^{J}=\mathrm{J}$, $\mathrm{H}^{\mathrm{J}}: \overline{\mathrm{U}} \times[0,1] \rightarrow \mathrm{K}(\mathrm{E})$ is a u.s.c. compact (see [1, Theorem 4.18]) map, $\mathrm{H}^{\mathrm{J}} \in \mathbf{A}(\overline{\mathrm{U}} \times[0,1], \mathrm{E})$ and $\Phi(\mathrm{x}) \cap$ $H_{t}^{J}(x)=\emptyset$ for $x \in \partial U$ and $t \in(0,1)$ (if $x \in \partial U$ and $t \in(0,1)$ then since $\left.J\right|_{\partial u}=\left.F\right|_{\partial u}$ we note that $\Phi(x) \cap H_{t}^{J}(x)=\Phi(x) \cap[t F(x)+(1-t) G(x)]$ ) so as a result $G \cong J$ (Definition 2.4) in $A_{\partial u}(\bar{U}, E)$ (i.e., (2.1) holds). [Note $E$ being a completely metrizable locally convex space can be replaced by any (Hausdorff) topological vector space $E$ which has the property that the closed convex hull of a compact set in $E$ is compact. In fact it is easy to see, if we argue differently, that all we need to assume is that $E$ is a topological vector space].

We now present the topological transversality theorem in a general setting. Assume

$$
\cong \text { in } A_{\partial u}(\bar{U}, E) \text { is an equivalence relation. }
$$

Theorem 2.10. Let $\mathrm{E}$ be a completely regular topological space, $\mathrm{U}$ an open subset of $\mathrm{E}$ and assume (2.2) holds. Suppose $\mathrm{F}$ and $\mathrm{G}$ are two maps in $\mathrm{A}_{\partial \mathrm{u}}(\overline{\mathrm{U}}, \mathrm{E})$ with $\mathrm{F} \cong \mathrm{G}$ in $\mathrm{A}_{\partial \mathrm{u}}(\overline{\mathrm{U}}, \mathrm{E})$. Then $\mathrm{F}$ is $\Phi$-essential in $\mathrm{A}_{\partial \mathrm{u}}(\overline{\mathrm{U}}, \mathrm{E})$ if and only if $\mathrm{G}$ is $\Phi$-essential in $\mathrm{A}_{\partial \mathrm{u}}(\overline{\mathrm{U}}, \mathrm{E})$.

Proof. Assume $G$ is $\Phi$-essential in $A_{\partial u}(\bar{U}, E)$. To show $F$ is $\Phi$-essential in $A_{\partial u}(\bar{U}, E)$ let $J \in A_{\partial u}(\bar{U}, E)$ with $\left.J\right|_{\partial u}=\left.F\right|_{\partial u}$ and $J \cong F$ in $A_{\partial u}(\bar{U}, E)$. Now since $F \cong G$ in $A_{\partial u}(\bar{U}, E)$ then (2.2) guarantees that $G \cong J$ in $A_{\partial u}(\bar{U}, E)$ i.e., (2.1) holds. Then Theorem 2.7 guarantees that $F$ is $\Phi$-essential in $A_{\partial u}(\bar{U}, E) . A$ similar argument shows that if $F$ is $\Phi$-essential in $A_{\partial u}(\bar{U}, E)$ then $G$ is $\Phi$-essential in $A_{\partial u}(\bar{U}, E)$.

Assume (2.2) holds. If $F$ and $G$ are maps in $A_{\partial u}(\bar{U}, E)$ with $\left.F\right|_{\partial u}=\left.G\right|_{\partial u}$ is $F \cong G$ in $A_{\partial u}(\bar{U}, E)$ ? We will discuss this now.

We assume the following conditions:

$E$ is a (Hausdorff) topological vector space and $U$ is convex

$$
\text { there exists a retraction } r: \bar{u} \rightarrow \partial u
$$

and

$$
\left\{\begin{array}{l}
\text { for any map } \Theta \in \mathbf{A}(\overline{\mathrm{U}}, \mathrm{E}) \text { and } \mathrm{f} \in \mathbf{C}(\overline{\mathrm{U}} \times[0,1], \overline{\mathrm{U}}) \\
\text { then } \Theta \circ f \in \mathbf{A}(\overline{\mathrm{U}} \times[0,1], \mathrm{E}) .
\end{array}\right.
$$

Remark 2.11. Note topological vector spaces are completely regular. Also if $E$ is an infinite dimensional Banach space and $\mathrm{U}$ is convex then (2.4) holds. Also note if $\mathbf{A}$ is closed under composition then (2.5) holds.

Let $r$ be in (2.4) and let $F$ and $G$ be maps in $A_{\partial u}(\bar{U}, E)$ with $\left.F\right|_{\partial u}=\left.G\right|_{\partial u}$. Consider the map $F^{\star}$ given by $F^{\star}(x)=F(r(x))$ for $x \in \bar{U}$. Note $F^{\star}(x)=G(r(x))$ for $x \in \bar{U}$ since $\left.F\right|_{\text {au }}=\left.G\right|_{\text {au }}$. Let

$$
H(x, \lambda)=G(2 \lambda r(x)+(1-2 \lambda) x)=G \circ j(x, \lambda) \text { for }(x, \lambda) \in \bar{U} \times\left[0, \frac{1}{2}\right]
$$

(here $j: \bar{U} \times\left[0, \frac{1}{2}\right] \rightarrow \bar{U}$ (note $\bar{U}$ is convex) is given by $j(x, \lambda)=2 \lambda r(x)+(1-2 \lambda) x$ ). Now $H: \bar{U} \times\left[0, \frac{1}{2}\right] \rightarrow$ $K(E)$ is a u.s.c. compact map. Also from (2.5) note $H \in \mathbf{A}\left(\bar{U} \times\left[0, \frac{1}{2}\right]\right.$, E) with $\Phi(x) \cap H_{\lambda}(x)=\emptyset$ for $x \in \partial U$ and $\lambda \in\left[0, \frac{1}{2}\right]$ (note if $x \in \partial U$ and $\lambda \in\left[0, \frac{1}{2}\right]$ then since $r(x)=x$ we have $\Phi(x) \cap H_{\lambda}(x)=\Phi(x) \cap G(x)$ ). Thus $\mathrm{G} \cong \mathrm{F}^{\star}$ in $A_{\partial \mathrm{u}}(\overline{\mathrm{U}}, \mathrm{E})$ (Definition 2.4). Similarly with

$$
\mathrm{Q}(x, \lambda)=\Phi((2-2 \lambda) \mathrm{r}(x)+(2 \lambda-1) x) \text { for }(x, \lambda) \in \overline{\mathrm{U}} \times\left[\frac{1}{2}, 1\right]
$$

we see that $F^{\star} \cong F$ in $A_{\partial u}(\bar{U}, E)$ (Definition 2.4). Combining gives $F \cong G$ in $A_{\partial u}(\bar{U}, E)$ (Definition 2.4).

In this situation we could replace Definition 2.6 with: 
Definition 2.12. Let $F \in A_{\partial u}(\bar{U}, E)$. We say $F$ is essential in $A_{\partial u}(\bar{U}, E)$ if for every map $J \in A_{\partial u}(\bar{U}, E)$ with $\left.\mathrm{J}\right|_{\partial u}=\left.\mathrm{F}\right|_{\partial u}$ there exists a $x \in \mathrm{U}$ with $\Phi(x) \cap \mathrm{J}(\mathrm{x}) \neq \emptyset$.

Now from Theorem 2.7 (in fact here the argument would be shorter since the map Q is not needed and the assumption $\Theta \circ f \in \mathbf{A}(\overline{\mathrm{U}} \times[0,1], \mathrm{E})$ is not needed in Definition 2.4) and Theorem 2.10 we have:

Theorem 2.13. Let $\mathrm{E}$ be a topological vector space, $\mathrm{U}$ an open convex subset of $\mathrm{E}$ and assume (2.2), (2.4) and (2.5) hold. Suppose $\mathrm{F}$ and $\mathrm{G}$ are two maps in $\mathrm{A}_{\partial \mathrm{u}}(\overline{\mathrm{U}}, \mathrm{E})$ with $\mathrm{F} \cong \mathrm{G}$ in $\mathrm{A}_{\partial \mathrm{u}}(\overline{\mathrm{U}}, \mathrm{E})$ (as in Definition 2.4). Then $\mathrm{F}$ is $\Phi$-essential (Definition 2.12) in $A_{\partial u}(\bar{U}, E)$ if and only if $G$ is $\Phi$-essential (Definition 2.12) in $A_{\partial u}(\bar{U}, E)$.

Remark 2.14.

(i). Suppose (2.4) and (2.5) hold and in addition assume

$$
\left\{\begin{array}{l}
\text { for any map } \Theta \in \mathbf{A}(\overline{\mathrm{U}}, \mathrm{E}) \text { then } \Theta(., \eta(.))=\Theta \circ f(., \eta(.)) \in \mathbf{A}(\overline{\mathrm{U}}, \mathrm{E}) \\
\text { for any continuous function } \eta: \overline{\mathrm{U}} \rightarrow[0,1] \text { with } \eta(\partial \mathrm{U})=0 \text { where } \\
f(x, t)=\operatorname{tr}(x)+(1-t) x, t \in[0,1], x \in \overline{\mathrm{U}}
\end{array}\right.
$$

Let $F$ and $G$ be maps in $A_{\partial u}(\bar{U}, E)$ with $\left.F\right|_{\partial u}=\left.G\right|_{\partial u}$. It is simple to adjust the proof above (use (2.6) instead of (2.5)) to establish $F \cong G$ in $A_{\partial u}(\bar{U}, E)$ (as in Remark 2.5). As a result we get immediately Theorem 2.13 (with (2.5) replaced by (2.6) and $\cong$ in $A_{\partial u}(\bar{U}, E)$ (Definition 2.4) replaced by $\cong$ in $A_{\partial u}(\bar{U}, E)$ (Remark 2.5)).

(ii). Let $F$ and $G$ be maps in $A_{\partial u}(\bar{U}, E)$ with $\left.F\right|_{\partial u}=\left.G\right|_{\partial u}$. Assume the following conditions:

$\mathrm{E}$ is a completely metrizable locally convex space

$$
\Phi(x) \cap[t F(x)+(1-t) G(x)]=\emptyset \text { for } x \in \partial U \text { and } t \in(0,1)
$$

and

$$
\left\{\begin{array}{l}
\eta(.) \mathrm{F}(.)+(1-\eta(.)) \mathrm{G}(.) \in \mathbf{A}(\overline{\mathrm{U}}, \mathrm{E}) \text { for any } \\
\text { continuous function } \eta: \overline{\mathrm{U}} \rightarrow[0,1] \text { with } \eta(\partial \mathrm{U})=0 .
\end{array}\right.
$$

Let $H(x, \lambda)=\lambda F(x)+(1-\lambda) G(x)$ for $(x, \lambda) \in \bar{U} \times[0,1]$. Note $H: \bar{U} \times[0,1] \rightarrow K(E)$ is a u.s.c. compact (see [1, Theorem 4.18]) map and by (2.9) note $\mathrm{H}(., \eta().) \in \mathbf{A}(\overline{\mathrm{U}}, \mathrm{E})$ for any continuous function $\eta: \overline{\mathrm{U}} \rightarrow$ $[0,1]$, and from (2.8) note $\Phi(x) \cap H_{t}(x)=\emptyset$ for $x \in \partial U$ and $t \in(0,1)$ so as a result $F \cong G$ in $A_{\partial u}(\bar{U}, E)$ (Remark 2.5). [Note (2.7) can be replaced by any topological vector space $E$ which has the property that the closed convex hull of a compact set in $E$ is compact]. As a result in this setting we get immediately Theorem 2.13 (with (2.3), (2.4), (2.5) replaced by (2.7), (2.8), (2.9) and $\cong$ in $A_{\partial u}(\bar{U}, E)$ (Definition 2.4) replaced by $\cong$ in $A_{\partial u}(\bar{U}, E)$ (Remark 2.5)).

Now we present an example of a $\Phi$-essential (Definition 2.12) map.

Example 2.15. Let $E$ be a (Hausdorff) topological space, $U$ an open subset of $E, \Phi \in B(E, E)$ (i.e., $\Phi \in \mathbf{B}(E, E)$ and $\Phi: E \rightarrow K(E)$ is a u.s.c. map) and $F \in A_{\partial u}(\bar{U}, E)$. Assume the following conditions hold:

$$
\begin{gathered}
\text { there exists a } x \in \overline{\mathrm{U}} \text { with } \Phi(x) \cap\{0\} \neq \emptyset \\
\text { there exists a retraction } r: E \rightarrow \overline{\mathrm{U}} \\
\Phi(x) \cap \lambda \mathrm{F}(\mathrm{x})=\emptyset \text { for } \mathrm{x} \in \partial \mathrm{U} \text { and } \lambda \in(0,1) \\
\left\{\begin{array}{l}
\text { for any continuous map } \mu: E \rightarrow[0,1] \text { with } \mu(E \backslash \mathrm{U})=0 \\
\text { and any map } \mathrm{J} \in \mathrm{A}_{\partial \mathrm{u}}(\overline{\mathrm{U}}, \mathrm{E}) \text { with }\left.\mathrm{J}\right|_{\partial \mathrm{u}}=\left.\mathrm{F}\right|_{\partial \mathrm{u}} \\
\text { there exists a } w \in \mathrm{E} \text { with } \Phi(w) \cap \mu(w) \mathrm{J}(\mathrm{r}(w)) \neq \emptyset
\end{array}\right.
\end{gathered}
$$

and

$$
\text { there is no } z \in \mathrm{E} \backslash \mathrm{U} \text { with } \Phi(z) \cap\{0\} \neq \emptyset \text {. }
$$


Then $F$ is $\Phi$-essential (Definition 2.12) in $A_{\partial u}(\bar{U}, E)$.

To see this let $\mathrm{J} \in \mathrm{A}_{\partial \mathrm{u}}(\overline{\mathrm{U}}, \mathrm{E})$ with $\left.\mathrm{J}\right|_{\partial \mathrm{u}}=\left.\mathrm{F}\right|_{\partial u}$. Now let

$$
\mathrm{K}=\{x \in \overline{\mathrm{U}}: \Phi(x) \cap \lambda \mathrm{J}(\mathrm{x}) \neq \emptyset \text { for some } \lambda \in[0,1]\} .
$$

Now $K \neq \emptyset$ (see (2.10)) is compact and $K \subseteq \bar{U}$. In fact $K \subseteq U$ from (2.12) (note if $x \in \partial U$ and $x \in K$ then for some $\lambda \in[0,1]$ we have $\emptyset \neq \Phi(x) \cap \lambda J(x)=\Phi(x) \cap \lambda F(x)$, a contradiction). Then there exists a continuous map $\mu: E \rightarrow[0,1]$ with $\mu(E \backslash U)=0$ and $\mu(K)=1$. Let $r$ be as in (2.11) and (2.13) guarantees that there exists a $x \in E$ with $\Phi(x) \cap \mu(x) J(r(x)) \neq \emptyset$. If $x \in E \backslash U$ then $\mu(x)=0$ so $\Phi(x) \cap\{0\} \neq \emptyset$, and this contradicts (2.14). Thus $x \in U$ so $\Phi(x) \cap \mu(x) J(x) \neq \emptyset$, so $x \in K, \mu(x)=1$ and consequently $\Phi(\mathrm{x}) \cap \mathrm{J}(\mathrm{x}) \neq \emptyset$.

Remark 2.16. It is very easy to extend the above ideas to the ( $L, T) \Phi$-essential maps in [6].

Now we consider a generalization of $\Phi$-essential maps, namely the $d-\Phi$-essential maps. Let $E$ be a completely regular topological space and $U$ an open subset of $E$. For any map $F \in A(\bar{U}, E)$ write $\mathrm{F}^{\star}=\mathrm{I} \times \mathrm{F}: \overline{\mathrm{U}} \rightarrow \mathrm{K}(\overline{\mathrm{U}} \times \mathrm{E})$, with $\mathrm{I}: \overline{\mathrm{U}} \rightarrow \overline{\mathrm{U}}$ given by $\mathrm{I}(\mathrm{x})=\mathrm{x}$, and let

$$
\mathrm{d}:\left\{\left(\mathrm{F}^{\star}\right)^{-1}(\mathrm{~B})\right\} \cup\{\emptyset\} \rightarrow \Omega
$$

be any map with values in the nonempty set $\Omega$ where $B=\{(x, \Phi(x)): x \in \bar{U}\}$.

Definition 2.17. Let $F \in A_{\partial u}(\bar{U}, E)$ and write $F^{\star}=I \times F$. We say $F^{\star}: \bar{U} \rightarrow K(\bar{U} \times E)$ is d- $\Phi$-essential if for every map $\mathrm{J} \in A_{\partial \mathrm{u}}(\overline{\mathrm{U}}, \mathrm{E})$ (write $\mathrm{J}^{\star}=\mathrm{I} \times \mathrm{J}$ ) with $\left.\mathrm{J}\right|_{\partial \mathrm{u}}=\left.\mathrm{F}\right|_{\partial \mathrm{u}}$ and $\mathrm{J} \cong \mathrm{F}$ in $A_{\partial \mathrm{u}}(\overline{\mathrm{U}}, \mathrm{E})$ we have that $\mathrm{d}\left(\left(\mathrm{F}^{\star}\right)^{-1}(\mathrm{~B})\right)=\mathrm{d}\left(\left(\mathrm{J}^{\star}\right)^{-1}(\mathrm{~B})\right) \neq \mathrm{d}(\emptyset)$.

Remark 2.18. If $\mathrm{F}^{\star}$ is $\mathrm{d}-\Phi-$-essential then

$$
\emptyset \neq\left(\mathrm{F}^{\star}\right)^{-1}(\mathrm{~B})=\{x \in \overline{\mathrm{U}}:(x, \mathrm{~F}(\mathrm{x})) \cap(x, \Phi(x)) \neq \emptyset\},
$$

so there exists a $x \in U$ with $(x, \Phi(x)) \cap(x, F(x)) \neq \emptyset$ (i.e., $\Phi(x) \cap F(x) \neq \emptyset)$.

Theorem 2.19. Let $\mathrm{E}$ be a completely regular topological space, $\mathrm{U}$ an open subset of $\mathrm{E}, \mathrm{B}=\{(\mathrm{x}, \Phi(\mathrm{x})): x \in \overline{\mathrm{U}}\}$, $\mathrm{d}$ is defined in (2.15), $\mathrm{F} \in \mathrm{A}_{\partial \mathrm{u}}(\overline{\mathrm{U}}, \mathrm{E})$ and $\mathrm{G} \in \mathrm{A}_{\partial \mathrm{u}}(\overline{\mathrm{U}}, \mathrm{E})$ (write $\mathrm{F}^{\star}=\mathrm{I} \times \mathrm{F}$ and $\left.\mathrm{G}^{\star}=\mathrm{I} \times \mathrm{G}\right)$. Suppose $\mathrm{G}^{\star}$ is $\mathrm{d}-\Phi-$ essential and

$$
\left\{\begin{array}{l}
\text { for any map } \mathrm{J} \in \mathrm{A}_{\partial \mathrm{u}}(\overline{\mathrm{U}}, \mathrm{E}) \text { with }\left.\mathrm{J}\right|_{\partial \mathrm{u}}=\left.\mathrm{F}\right|_{\partial \mathrm{u}} \text { and } \\
\mathrm{J} \cong \mathrm{F} \text { in } \mathrm{A} \mathrm{\partial u}(\overline{\mathrm{U}}, \mathrm{E}) \text { we have } \mathrm{G} \cong \mathrm{J} \text { in } \mathrm{A} \partial \mathrm{u}(\overline{\mathrm{U}}, \mathrm{E}) \\
\text { and } \mathrm{d}\left(\left(\mathrm{F}^{\star}\right)^{-1}(\mathrm{~B})\right)=\mathrm{d}\left(\left(\mathrm{G}^{\star}\right)^{-1}(\mathrm{~B})\right)
\end{array}\right.
$$

Then $\mathrm{F}^{\star}$ is $\mathrm{d}-\Phi-$ essential.

Proof. Without loss of generality assume $\cong$ in $A_{\partial u}(\bar{U}, E)$ is as in Definition 2.4. Consider any map $J \in$ $A_{\partial u}(\bar{U}, E)$ (write $\left.J^{\star}=I \times J\right)$ with $\left.J\right|_{\partial u}=\left.F\right|_{\partial u}$ and $J \cong F$ in $A_{\partial u}(\bar{U}, E)$. From (2.16) there exists a u.s.c. compact map $H^{J}: \bar{U} \times[0,1] \rightarrow K(E)$ with $H^{J} \in \mathbf{A}(\bar{U} \times[0,1], E), \Phi(x) \cap H_{t}^{J}(x)=\emptyset$ for any $x \in \partial U$ and $t \in(0,1)$ (here $\left.H_{t}^{J}(x)=H^{J}(x, t)\right), H_{0}^{J}=G, H_{1}^{J}=J$ and $d\left(\left(F^{\star}\right)^{-1}(B)\right)=d\left(\left(G^{\star}\right)^{-1}(B)\right)$. Let $\left(\mathrm{H}^{\mathrm{J}}\right)^{\star}: \overline{\mathrm{U}} \times[0,1] \rightarrow \mathrm{K}(\overline{\mathrm{U}} \times \mathrm{E})$ be given by $\left(\mathrm{H}^{\mathrm{J}}\right)^{\star}(\mathrm{x}, \mathrm{t})=\left(\mathrm{x}, \mathrm{H}^{\mathrm{J}}(\mathrm{x}, \mathrm{t})\right)$ and let

$$
\mathrm{K}=\left\{x \in \overline{\mathrm{U}}:(x, \Phi(x)) \cap\left(\mathrm{H}^{\mathrm{J}}\right)^{\star}(x, \mathrm{t}) \neq \emptyset \text { for some } \mathrm{t} \in[0,1]\right\} .
$$

Now $K \neq \emptyset$ is closed, compact and $K \cap \partial U=\emptyset$ so since $E$ is Tychonoff there exists a continuous map $\mu: \overline{\mathrm{U}} \rightarrow[0,1]$ with $\mu(\partial \mathrm{U})=0$ and $\mu(\mathrm{K})=1$. Let $\mathrm{R}(\mathrm{x})=\mathrm{H}^{\mathrm{J}}(\mathrm{x}, \mu(\mathrm{x}))$ and write $\mathrm{R}^{\star}=\mathrm{I} \times \mathrm{R}$. Now as in Theorem 2.7, $R \in A_{\partial u}(\bar{U}, E)$ with $\left.R\right|_{\partial u}=\left.G\right|_{\partial u}$ and $R \cong G$ in $A_{\partial u}(\bar{U}, E)$. Since $G^{\star}$ is $d-\Phi$-essential then

$$
\mathrm{d}\left(\left(\mathrm{G}^{\star}\right)^{-1}(\mathrm{~B})\right)=\mathrm{d}\left(\left(\mathrm{R}^{\star}\right)^{-1}(\mathrm{~B})\right) \neq \mathrm{d}(\emptyset) .
$$


Now since $\mu(K)=1$ we have

$$
\begin{aligned}
\left(R^{\star}\right)^{-1}(B) & =\left\{x \in \overline{\mathrm{U}}:(x, \Phi(x)) \cap\left(x, \mathrm{H}^{\mathrm{J}}(x, \mu(x))\right) \neq \emptyset\right\} \\
& =\left\{x \in \overline{\mathrm{U}}:(x, \Phi(x)) \cap\left(x, \mathrm{H}^{\mathrm{J}}(\mathrm{x}, 1)\right) \neq \emptyset\right\}=\left(\mathrm{J}^{\star}\right)^{-1}(\mathrm{~B}),
\end{aligned}
$$

so from (2.17) we have $d\left(\left(G^{\star}\right)^{-1}(B)\right)=d\left(\left(J^{\star}\right)^{-1}(B)\right) \neq d(\emptyset)$. Now combine with the above and we have $d\left(\left(F^{\star}\right)^{-1}(B)\right)=d\left(\left(J^{\star}\right)^{-1}(B)\right) \neq d(\emptyset)$.

Note again it is simple to adjust the proof in Theorem 2.19 if we use $\cong$ in $A_{\partial u}(\bar{U}, E)$ from Remark 2.5.

Theorem 2.20. Let $\mathrm{E}$ be a completely regular topological space, $\mathrm{U}$ an open subset of $\mathrm{E}, \mathrm{B}=\{(\mathrm{x}, \Phi(\mathrm{x})): x \in \overline{\mathrm{U}}\}$, $\mathrm{d}$ is defined in (2.15) and assume (2.2) holds. Suppose $\mathrm{F}$ and $\mathrm{G}$ are two maps in $\mathrm{A}_{\partial \mathrm{u}}(\overline{\mathrm{U}}, \mathrm{E})$ (write $\mathrm{F}^{\star}=\mathrm{I} \times \mathrm{F}$ and $\left.\mathrm{G}^{\star}=\mathrm{I} \times \mathrm{G}\right)$ and $\mathrm{F} \cong \mathrm{G}_{\text {in }} \mathrm{A}_{\partial \mathrm{u}}(\overline{\mathrm{U}}, \mathrm{E})$. Then $\mathrm{F}^{\star}$ is $\mathrm{d}-\Phi-$ essential if and only if $\mathrm{G}^{\star}$ is $\mathrm{d}-\Phi-$-essential.

Proof. Without loss of generality assume $\cong$ in $A_{\partial u}(\bar{U}, E)$ is as in Definition 2.4. Assume $G^{\star}$ is $d-\Phi-$ essential. Let $\mathrm{J} \in A_{\partial \mathrm{u}}(\overline{\mathrm{U}}, \mathrm{E})$ (write $\mathrm{J}^{\star}=\mathrm{I} \times \mathrm{J}$ ) with $\left.\mathrm{J}\right|_{\partial \mathrm{u}}=\left.\mathrm{F}\right|_{\partial \mathrm{u}}$ and $\mathrm{J} \cong \mathrm{F}$ in $A_{\partial \mathrm{u}}(\overline{\mathrm{U}}, \mathrm{E})$. If we show (2.16) then $F^{\star}$ is $d-\Phi$-essential from Theorem 2.19. Now (2.2) implies that $G \cong J$ in $A_{\partial u}(\bar{U}, E)$. To complete (2.16) we need to show $d\left(\left(F^{\star}\right)^{-1}(B)\right)=d\left(\left(G^{\star}\right)^{-1}(B)\right)$. We will follow the argument in Theorem 2.19. Note since $G \cong F$ in $A_{\partial u}(\bar{U}, E)$ let $H: \bar{U} \times[0,1] \rightarrow K(E)$ be a u.s.c. compact map with $H \in \mathbf{A}(\overline{\mathrm{U}} \times[0,1], E), \Phi(x) \cap H_{t}(x)=\emptyset$ for any $x \in \partial U$ and $t \in(0,1)$ (here $\left.H_{t}(x)=H(x, t)\right), H_{0}=G$ and $\mathrm{H}_{1}=\mathrm{F}$. Let $\mathrm{H}^{\star}: \overline{\mathrm{U}} \times[0,1] \rightarrow \mathrm{K}(\overline{\mathrm{U}} \times \mathrm{E})$ be given by $\mathrm{H}^{\star}(\mathrm{x}, \mathrm{t})=(\mathrm{x}, \mathrm{H}(\mathrm{x}, \mathrm{t}))$ and let

$$
K=\left\{x \in \bar{U}:(x, \Phi(x)) \cap H^{\star}(x, t) \neq \emptyset \text { for some } t \in[0,1]\right\} .
$$

Now $K \neq \emptyset$ and there exists a continuous map $\mu: \bar{U} \rightarrow[0,1]$ with $\mu(\partial U)=0$ and $\mu(K)=1$. Let $R(x)=H(x, \mu(x))$ and write $R^{\star}=I \times R$. Now $R \in A_{\partial u}(\bar{U}, E)$ with $\left.R\right|_{\partial u}=\left.G\right|_{\partial u}$ and $R \cong G$ in $A_{\partial u}(\bar{U}, E)$ so since $G^{\star}$ is $d-\Phi$-essential then $d\left(\left(G^{\star}\right)^{-1}(B)\right)=d\left(\left(R^{\star}\right)^{-1}(B)\right) \neq d(\emptyset)$. Now since $\mu(K)=1$ we have

$$
\begin{aligned}
\left(R^{\star}\right)^{-1}(B) & =\{x \in \overline{\mathrm{U}}:(x, \Phi(x)) \cap(x, H(x, \mu(x))) \neq \emptyset\} \\
& =\{x \in \overline{\mathrm{U}}:(x, \Phi(x)) \cap(x, H(x, 1)) \neq \emptyset\}=\left(F^{\star}\right)^{-1}(B),
\end{aligned}
$$

so $d\left(\left(F^{\star}\right)^{-1}(B)\right)=d\left(\left(G^{\star}\right)^{-1}(B)\right)$.

Note again it is simple to adjust the proof in Theorem 2.20 if we use $\cong$ in $A_{\partial u}(\bar{U}, E)$ from Remark 2.5. Remark 2.21. It is very easy to extend the above ideas to the $(L, T) d-\Phi$-essential maps in [7].

\section{References}

[1] C. D. Aliprantis, K. C. Border, Infinite-Dimensional Analysis, Studies in Economic Theory, Springer-Verlag, Berlin, (1994). 2, 2.14

[2] R. Engelking, General Topology, PWN-Polish Scientific Publishers, Warszawa, (1977). 2

[3] L. Górniewicz, Topological fixed point theory of multivalued mappings, Kluwer Academic Publ., Dordrecht, (1999). 1

[4] A. Granas, Sur la méthode de continuité de Poincaré, C. R. Acad. Sci. Paris Sér. A-B, 282 (1976), 983-985. 1

[5] W. Kryszewski, Topological and approximation methods of degree theory of set-valued maps, Dissertationes Math. (Rozprawy Mat.), 336 (1994), 101 pages. 1

[6] D. O'Regan, Generalized coincidence theory for set-valued maps, J. Nonlinear Sci. Appl., 10 (2017), 855-864. 1, 2.16

[7] D. O'Regan, Topological transversality principles and general coincidence theory, An. tiin. Univ. "Ovidius" Constana Ser. Mat., 25 (2017), 159-170. 2.21

[8] R. Precup, On the topological transversality principle, Nonlinear Anal., 20 (1993), 1-9. 1 